\title{
A Taxonomy of Preferences for Physically Assistive Robots*
}

\author{
Gerard Canal ${ }^{1}$, Guillem Alenyà and Carme Torras
}

\begin{abstract}
Assistive devices and technologies are getting common and some commercial products are starting to be available. However, the deployment of robots able to physically interact with a person in an assistive manner is still a challenging problem. Apart from the design and control, the robot must be able to adapt to the user it is attending in order to become a useful tool for caregivers. This robot behavior adaptation comes through the definition of user preferences for the task such that the robot can act in the user's desired way. This article presents a taxonomy of user preferences for assistive scenarios, including physical interactions, that may be used to improve robot decision-making algorithms. The taxonomy categorizes the preferences based on their semantics and possible uses. We propose the categorization in two levels of application (global and specific) as well as two types (primary and modifier). Examples of real preference classifications are presented in three assistive tasks: feeding, shoe fitting and coat dressing.
\end{abstract}

\section{INTRODUCTION}

Assistive Robotics, systems able to support people with physical and cognitive disabilities, are society's answer to help the maintenance of the workforce in the health care sector against the growth of aging population. While rehabilitation robotics have received more attention from the research community, the field is going towards the development of autonomous assistive systems to be employed by non-technical users. Socially Assistive Robots (SAR) [1] are those that provide assistance by means of social interaction to guide processes of rehabilitation, learning and convalescence. On the other hand, Physically Assistive Robots (PAR) are the ones which provide assistance by means of physical interaction, helping users to perform activities such as eating, dressing and grooming [2], [3].

The fact of considering physical interactions between the human and the robot introduces a new set of requirements in terms of robot action execution, and thus, also in terms of user preferences. There are hundreds of ways of assisting a person to perform Activities of the Daily Living (ADLs), along with other subtleties that human caregivers take into account. Caregivers, knowing the users and interacting with them, adapt the assistance to suit every individual's needs and preferences effectively making the task more pleasant for the patients. However, the loss of independence to perform

\footnotetext{
*This research is partially funded by the Spanish Ministry of Economy and Competitiveness under project TIN2014-58178-R and by the ERANet CHISTERA project I-DRESS PCIN-2015-147. Gerard Canal is also supported by the Spanish Ministry of Education, Culture and Sport via a FPU doctoral grant FPU15/00504.

${ }^{1}$ G. Canal, G. Alenyà and C. Torras are with Institut de Robòtica i Informàtica Industrial, CSIC-UPC, C/ Llorens i Artigas 4-6, 08028 Barcelona, Spain. $\{$ gcanal, galenya, torras\}eiri.upc.edu
}

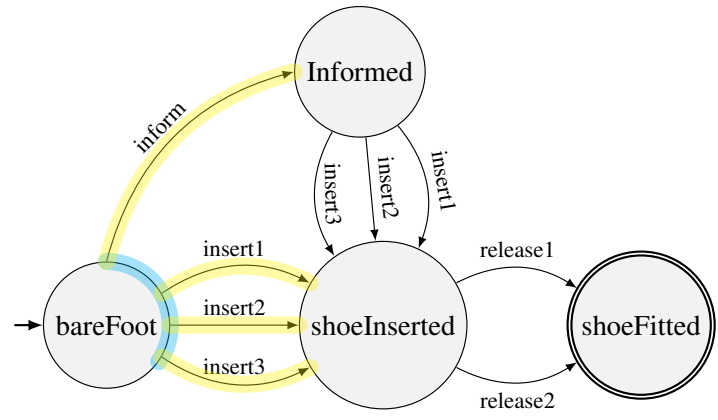

Fig. 1: Graphical example of decision-making (blue) and configuration preferences (yellow): in this action-sequence flow for the shoe fitting task, represented as a FSA, decisionmaking preferences aid to choose among alternative paths, while the configuration ones help to tune action parameters.

ADLs [4] results in great impact in the patient's psychological well-being, with feelings of burden and guilt to those close to them [5]. Thus, robots capable of physically assisting users in order to enable them to perform such tasks without the need of another human may have a considerable impact in the modern society [6]. Still, to successfully empower the users and ease the employment of robotic systems, such mechanical assistants should be able to cope with user abilities and preferences, just as human caregivers do.

In this paper, we answer the question of "how can we define and classify preferences?" in the context of PARs by defining a taxonomy of user preferences for HumanRobot Interaction applications ${ }^{2}$, putting special emphasis on physically assistive scenarios in which the inclusion of these preferences will make a difference. The taxonomy will ease the definition and classification of the preferences which, written in non-technical language, facilitate the inclusion of caregivers in the loop of assistive applications design. Moreover, the taxonomy will also be useful to implement preference-based applications that take into account the different categories. This customization of the applications will allow the adaptation of the robot's autonomy from a simple tool to a shared-autonomy system or a fully autonomous robotic assistant.

When taking into account possible contacts between the person and the robot, we have identified two loops in the execution of actions: a higher-level decision making in terms of finding a sequence of symbolic actions, and a lower-level one to perform the task. We observe that preference specification

\footnotetext{
${ }^{2}$ The taxonomy could also be used to define preferences in a more generic assistance scenario with a human caregiver and a patient, but in this paper we are mainly interested in the HRI scenario.
} 
in the former has received more attention of the community, while the latter is less explored as it requires the grounding of the involved symbols. In the presented taxonomy, this has been translated into preferences that permit guiding action selection (named decision-making preferences) and those that define how the selected operators are executed (named configuration preferences).

In Figure 1 we exemplify different states in a shoe fitting task along with some state transition actions. The robot has three shoe insertion actions and two shoe release operations available, and can inform the user before inserting the shoe. The selection of the action (whether it has to inform or which insertion should it use) is based on the decisionmaking preferences (marked in blue), while the configuration preferences define how -with which parameters- the selected action is to be performed (depicted in yellow).

\section{RELATED WORK}

Preferences are a central problem in decision making. Recently, a comprehensive survey has been published that reviews the different alternatives for modeling and using preferences in Artificial Intelligence [7]. Preference Based Planning (PBP) [8] is an extension of classical planning where a criterion is provided to select one plan among other valid plans based on user preferences. Hierarchical Task Networks (HTN) have been also used to encode user preferences [9]. In HTNs, a hierarchy of non-primitive actions is provided along with a set of methods to decompose them into primitive actions. The manual construction of HTNs indirectly encodes the user preferences, but is complex, error prone, and preferences are not always explicitly stated. Unfortunately, these works do not consider particular problems that appear in robotics and physical interaction.

The planning community often use the Planning Domain Description Language (PDDL) to describe planning domains. PDDL3 [10] was the first to define the preference construct, which allows to describe three types of preferences. The temporally extended preferences consist in desirable temporal relationships, the precondition preferences are atemporal formulae that should hold true in the state in which an action is to be performed, and the simple -also called goalpreferences are conditions that should hold in the final state. Sohrabi et al. [11] address the generation of preferred plans by extending the PDDL3 language to handle preferences over HTN constructs, supporting desires on how the tasks are decomposed.

Son and Pontelli [12] divide the preferences in different categories: preferences about a state define the preferred properties to hold in a state; preferences about an action describe actions that are preferred; preferences about a trajectory define preferred properties over sequences of actions; finally, multi-dimensional preferences consist in a set of preferences and an ordering among them. The authors introduce the language $\mathcal{P} \mathcal{P}$ for planning preferences specification and subdivide the preferences in basic desires, atomic preferences and general preferences. Although this categorization is suitable for planning and other problem solving tasks, we find it is not sufficient to define a set of preferences for physical interactions [13]. In our case, we propose a hierarchical taxonomy in which preferences are categorized by function and type.

A taxonomy for Human-Robot Interaction was proposed by Yanco and Drury [14] that allows to express elements such as: the social nature of the task, its type, the robot morphology and the interaction roles between teams of humans and robots. The taxonomy, however, does not include elements related to the preferences of the user but rather focuses on the interaction scenario.

Krauss and Arbanowski [15] build a social preference ontology to tackle typical issues of recommender systems, such as the cold start and the sparsity problems. The ontology represents topics the user is interested in along with a numerical score, and is filled up with information mined from social networks. Being task-specific, these ontologies do not suit our assistive robotics scenario as they lack the semantics specific to the personal satisfaction domain.

Bastemeijer et al. [16] define a taxonomy of the concepts patients value in health care based on a thorough literature review of several studies. They define three top-level categories: patient and personal context, the characteristics of the professional and the interaction between the patient and the professional. The key elements inside these categories are: uniqueness, autonomy, compassion, professionalism, responsiveness, partnership and empowerment. Although the elements they define could well suit our scenario, their concepts relate to general health care and patient's feelings, while our proposal is focused on defining key aspects of the behavior of the (robotic) assistant in the physical assistance environment.

A framework for levels of autonomy (LoA) is proposed alongside with a 10-point taxonomy in [17]. The taxonomy specifies each level of autonomy from the perspective of the human-robot interactions and the roles they play, and divides the HRI variables in robot-related, social, and humanrelated. We can link this definition of LoA to our proposal of preference categorization, as the set of values of the preferences can be used to determine the resulting LoA: from "shared control with human initiative" or "shared control with robot initiative" to "full autonomy".

Regarding social aspects of interaction, Peng et al. [18] propose a hierarchical taxonomy for robotic dance. Shim and Arkin [19] define a taxonomy of robot deception for HRI contexts. Wiltshire et al. [20] propose a taxonomy of social signals from an interdisciplinary point of view. They categorize five social cues that can be extracted to predict social signals. However, although they may look similar in some aspects, their taxonomy is presented to categorize the human behavior's rather than the robot's as we intend in our proposal.

Fong et al. [21] present a taxonomy of design methods, system components and applications for socially interactive robots, but preferences were not yet included. There exist other general robotics ontologies, such as KnowRob [22], which provide robots with knowledge of the environment, 
the actions, the tasks and mathematical concepts, and may be extended with information about preferences.

The concept of preference tends to be quite applicationspecific, as the reviewed works show. Though there are taxonomies for social robotics, they are still not enough to categorize the user preferences regarding the robot's behavior, which we are dealing with in this work. More specifically, we define the preferences for assistive tasks in robotics, taking inspiration from the commented works such as [21], and directly including the Big Five personality traits ontology [23] where personality is described based on five traits: extraversion, agreeableness, conscientiousness, neuroticism and openness.

\section{A TAXONOMY OF PREFERENCES FOR ASSISTIVE HUMAN-ROBOT INTERACTION}

In this section, we present a hierarchical taxonomy of user preference categories designed for Human-Robot Interaction applications, with emphasis on assistive scenarios where the robot aids users facing difficulties to perform Activities of Daily Living (ADLs). The taxonomy has been developed based on previous experiences, intuition and comments from health care providers and potential users, and motivated by the need to classify preferences in order to use them. To maintain generality, we do not distinguish between preferences and user constraints (such as mobility issues), as the latter can be expressed as a preference. For instance, a user who cannot move the right arm will "prefer" not to use this arm. Encoding impairments as preferences allows us to present a less limited taxonomy.

To begin with, we define two types of preferences: the primary preferences and the modifier ones. The former are preferences that are directly applicable, while the latter are used to accompany the primary preferences and modify them, effectively conditioning their applicability.

The proposed taxonomy is divided into two main category groups: the decision-making and the configuration preferences (Fig. 2). The Decision-making (DM) preferences are those that help the robot to choose between the different actions that it can execute at a given moment, provided that they all lead to the final goal (see left branch of Fig. 2). DM preferences are in turn divided into two categories, which are again subdivided into more fine-grained preference types:

- Communication preferences regulate the desired amount of different kinds of interaction with the robot. They are subdivided as:

- Information providing: whether the robot should inform regarding each performed action or should omit unimportant information.

- Information obtaining: define if the user prefers the robot to inquire about missing information or either it should try to infer it from other sources.

- Petitions: state if the robot should ask the user to perform some action (such as repositioning himself to ease the solution of a task) or if the robot should risk to accomplish the task without bothering the user, provided that no safety issue can arise at any moment.

- Contextual preferences define how the robot's behavior may change depending on the execution context, it being defined as the user's environment, place and time. We define four subcategories:

- Task: state preferences that have implications about the task that is being performed. They may define general user constraints (such as limited right arm mobility) or simple preferences such as how is more comfortable to scoop the spoon when eating. They may be subdivided in:

* Cognitive preferences related to cognitive disabilities of the user.

* Motor constraints of the user which may limit the task.

* Personal tastes are other personal needs and desires.

- Environment: preferences regarding the execution setting. They are mainly modifier preferences (see Fig. 2) that accompany primary preferences and limit their application range. We propose a subdivision in three categories:

* Moment: define the time of the day in which the task is executed, thus preferences may vary depending on, for instance, whether the task is performed in the morning or at night as the state of the person may be different regarding tiredness and mood.

* Company: preferences concerning the personal elements that are in the environment. User preferences with the robot may be different when a caregiver is also assisting the user in contrast to when a family member is doing so. Besides, the preferences will be others when the user is alone with the robot, given that he may need more support in that case.

* Situation: the preferences related to the location of the user. User preferences may change depending on where the task is being executed. For instance, it may not be the same to fit a shoe while seating on the bed than fitting a slipper while resting on the coach.

On the other hand, configuration (C) preferences (see right branch of Fig. 2) are those preferences that define how an action is to be performed. They are used to tune the parameters of the actions rather than choosing the action sequence that is going to be executed to solve the task. Configuration preferences are also divided into two categories:

- Physical preferences define the physical properties of the (physical) actions. These include:

- Proxemic: relate to the spatial requirements of the user, the robot and the task.

- Temporal: define temporal requirements of the user and the task. For instance, the user may prefer to not have the foot lifted for more than one minute. 


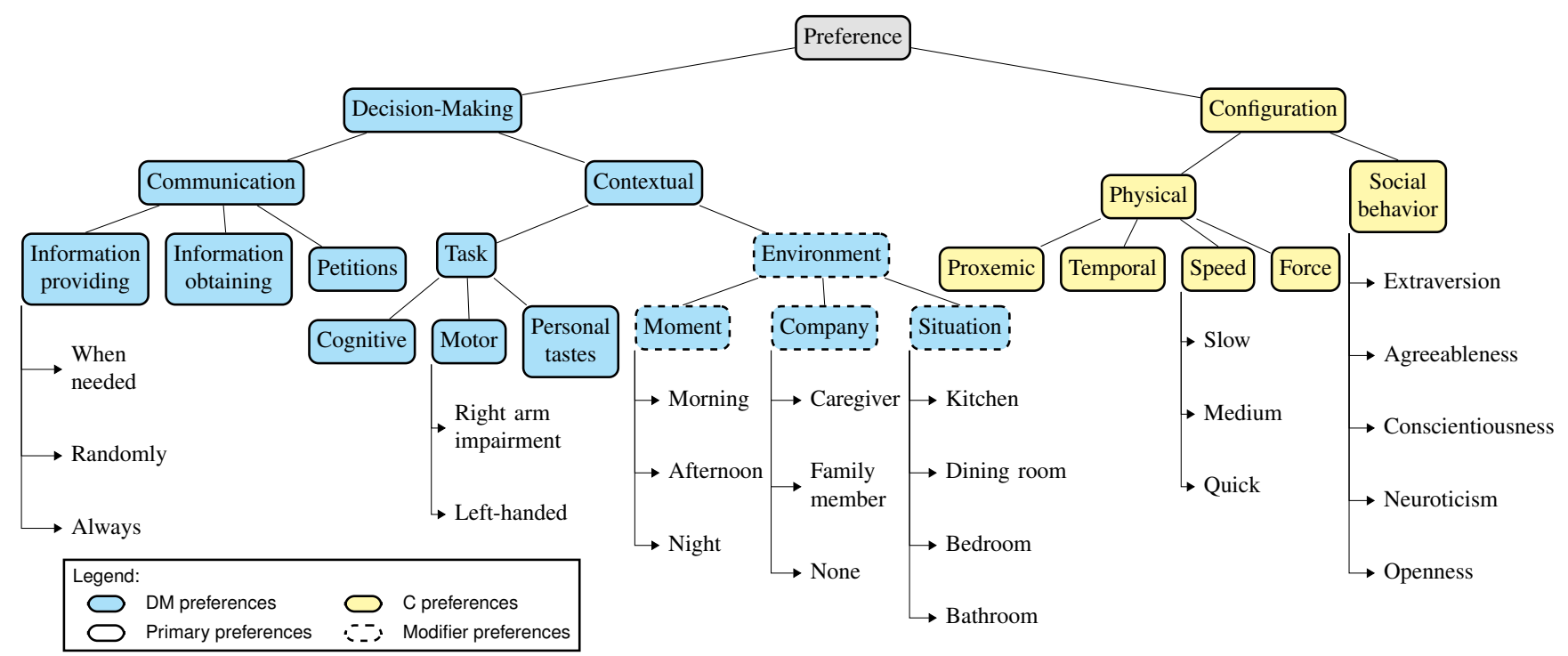

Fig. 2: Preference taxonomy for assistive physical Human-Robot Interaction.

- Speed: specify how quick or slow the user wants the robot to move. This relates to the feeling of safety, as the user may get scared if the robot makes sudden fast movements, but may also get impatient when the movements are too slow.

- Force: some tasks, such as shoe fitting, require pressure against some body parts. The applied force may be limited based on user desires and abilities.

- Social behavior preferences somehow characterize the robot's personality. Following the definition of [21], here we link our taxonomy with the Big Five personality trait taxonomy [23] which describes personality in terms of five traits (extraversion, agreeableness, conscientiousness, neuroticism and openness). With these preferences, the user can define personality-based items. Other elements that could be included as social behavior preferences are the kind of voice, tone, formality level, prose and detail level of the interactive acts.

The proposed taxonomy allows us to define user preferences for Human-Robot interaction tasks, and more specifically, for Physically Assistive Robots (PAR) to help elderly and handicapped people. However, future assistive robots should be able to perform more than one assistive task. Thus, there is a lot of redundancy when instantiating the preference taxonomy for every specific task. For instance, a user who prefers the robot to move slowly while fitting a shoe will probably prefer it to be slow when dressing a coat. Or he may have reduced mobility in his right arm, which implies that the user will need special assistance to perform any activity involving this arm. To solve this redundancy, and to ease the description of the preferences, we propose to define them in a two-level manner:

- Global preferences, are those that are applicable to most tasks. They define generic user preferences and personal constraints which may be used in any setup.

- Specific preferences define activity-related preferences.
They only apply to certain cases and during the execution of specific tasks.

Note that we do not restrict the possibility of specific preferences including elements that are already present in the global preferences, and they may even be in conflict by stating opposing elements. We tackle this by setting an importance level in which specific preferences take over the global preferences when a conflict arises. In this way, a specific preference of the same kind of a global preference overrides it, allowing the user to have task-specific tastes without the need of repeating a general desire for every task.

Given that PAR are actually touching the human users, safety-related preferences are not taken into consideration. We believe that a Physically Assistive Robot must be safe out-of-the-box, and the user shouldn't be able to modify the safety level. Though strict, this restriction leads to the development of intrinsically safe systems which must not try to perform any action that may potentially hurt a person cohabiting the robot's environment.

\section{APPLICATION EXAMPLES}

This section illustrates how the taxonomy can be instantiated for different Physically Assistive tasks as the ones shown in Figure 3, consisting in feeding, coat dressing and shoe fitting. To do so, we will define a fictional persona [24] and instantiate her preferences:
- Aunt Mery is an 80 years old granny who lives alone. Although she's healthy, she is suffering from lower back pain and recovering from a fracture in her right arm. Due to these issues, she needs help to carry out some ADLs such as putting shoes on, dressing a jacket to go to the therapist or eating. Thus, a Physically Assistive Robot will help her to maintain some autonomy while she is recovering.

Table I shows the global preferences, which are applicable to any task. Tables II, III and IV show the (specific) pref- 


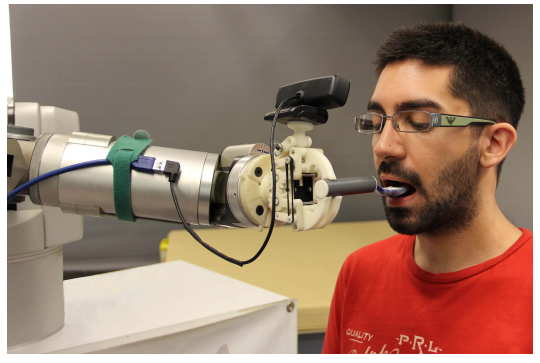

(a) Feeding assistance.

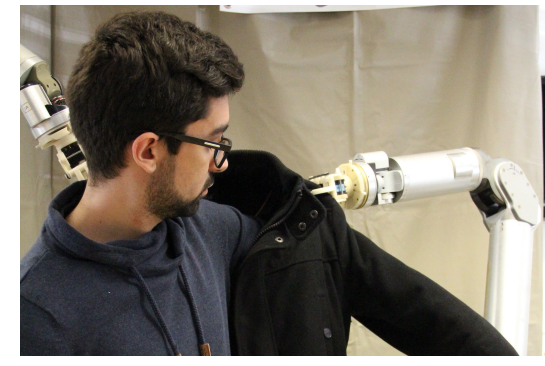

(b) Coat dressing assistance.

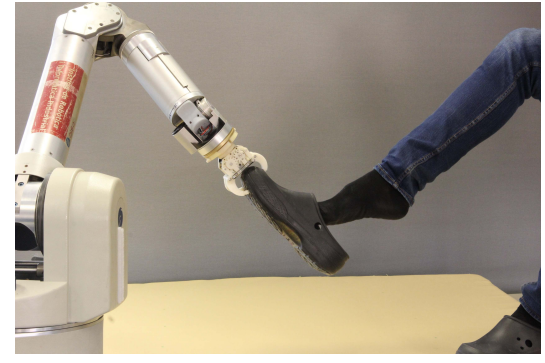

(c) Shoe fitting assistance.

Fig. 3: Physically Assistive Robot (PAR) application examples.

erences for the coat dressing, shoe fitting and feeding tasks, respectively. The "textual definition" column represents what Aunt Mery would say to describe each preference. The specific preferences that override a global preference are marked with an asterisk $(*)$. Note that when two specific preferences collide due to an additional modifier preference, the modified preference precedes the other ones, provided that the modifying condition holds. For instance, Mery prefers to eat slowly in the morning, though a medium velocity (global preference, Table I) is better for any other time of the day (Table IV). Also, she cannot wait for more than thirty seconds with the foot lifted when she is alone, but she can hold it up for a minute when there's a family member helping her (Table III). When dressing a coat, a normal force is fine during most of the task (Table II), however she prefers the robot to apply less force when the injured right arm is being dressed. Nevertheless, she prefers to start with the left foot when fitting a shoe (Table III), but the right arm is chosen when dressing a jacket (Table II). The tables demonstrate how, even though some assistive tasks may look similar, the taxonomy allows to freely define different preferences regarding the same task-depending aspects.

\section{CONCLUSIONS}

We have presented a taxonomy of preferences for assistive scenarios. The taxonomy allows to categorize the preferences the user may have regarding the behavior of the assistant during the task that is to be carried out. The preferences are first divided into the decision-making and configuration categories, depending on whether they are used to choose which action to perform or configure the action that is being executed. Moreover, some of the preferences, called "modifier preferences", are used to modify the applicability of other preferences. Finally, redundancies in the expression of the preferences are avoided with the definition of global preferences and task-specific preferences. The taxonomy will be useful to define user preferences in a structured way, which can then be used for assistive robotics applications, and more specifically, for those entailing physical interaction.

The taxonomy has been exemplified using a user persona whose preferences have been explicitly defined for the tasks of feeding and dressing. However, we have not entered into details about implementation of the preferences nor the preference elicitation and inference, which are currently ongoing work.

\section{ACKNOWLEDGMENT}

The authors thank Helena Parnaud for fruitful discussions.

\section{REFERENCES}

[1] D. Feil-Seifer and M. J. Matarić, "Defining socially assistive robotics," in 9th Int. Conf. on Rehabilitation Robotics, June 2005, pp. 465-468.

[2] G. Canal, G. Alenyà, and C. Torras, "Personalization Framework for Adaptive Robotic Feeding Assistance," in 8th Int. Conf. on Social Robotics (ICSR). Springer International Publishing, 2016, pp. 22-31.

[3] G. Chance, A. Camilleri, B. Winstone, P. Caleb-Solly, and S. Dogramadzi, "An assistive robot to support dressing - strategies for planning and error handling," in 6th IEEE Int. Conf. on Biomedical Robotics and Biomechatronics, June 2016, pp. 774-780.

[4] M. A. Gignac and C. Cott, "A conceptual model of independence and dependence for adults with chronic physical illness and disability," Social Science \& Medicine, vol. 47, no. 6, pp. 739-753, 91998.

[5] A. M. Williams, G. Christopher, and E. Jenkinson, "The psychological impact of dependency in adults with chronic fatigue syndrome/myalgic encephalomyelitis: A qualitative exploration," Health Psychol., 2016.

[6] T. L. Chen, M. Ciocarlie, S. Cousins, P. M. Grice, K. Hawkins, Kaijen Hsiao, C. C. Kemp, Chih-Hung King, D. a. Lazewatsky, A. E. Leeper, Hai Nguyen, A. Paepcke, C. Pantofaru, W. D. Smart, and L. Takayama, "Robots for humanity: using assistive robotics to empower people with disabilities," IEEE Robot. Autom. Mag., vol. 20, pp. 30-39, 2013.

[7] G. Pigozzi, A. Tsoukiàs, and P. Viappiani, "Preferences in artificial intelligence," Ann. Math. Artif. Intell., vol. 77, pp. 361-401, 2016.

[8] J. A. Baier and S. McIlraith, "Planning with preferences," AI Magazine, vol. 29, no. 4, pp. 25-36, 2008.

[9] N. Li, W. Cushing, S. Kambhampati, and S. Yoon, "Learning Probabilistic Hierarchical Task Networks as Probabilistic Context-Free Grammars to Capture User Preferences," ACM Transactions on Intelligent Systems and Technology, vol. 5, no. 2, pp. 1-32, 42014.

[10] A. E. Gerevini, P. Haslum, D. Long, A. Saetti, and Y. Dimopoulos, "Deterministic planning in the fifth international planning competition: PDDL3 and experimental evaluation of the planners," Artificial Intelligence, vol. 173 , no. 56, pp. $619-668,2009$.

[11] S. Sohrabi, J. A. Baier, and S. A. McIlraith, "HTN planning with preferences," Int. Joint Conf. on AI (IJCAI), pp. 1790-1797, 2009.

[12] T. C. Son and E. Pontelli, "Planning with preferences using logic programming," Theory and Practice of Logic Programming, vol. 6, no. 05, pp. 559-607, 92006.

[13] B. D. Argall and A. G. Billard, "A survey of Tactile HumanRobot Interactions," Robot. Auton. Syst., vol. 58, no. 10, pp. 1159-1176, 2010.

[14] H. Yanco and J. Drury, "Classifying human-robot interaction: an updated taxonomy," in Int. Conf. on Systems, Man and Cybernetics, vol. 3. IEEE, 2004, pp. 2841-2846.

[15] C. Krauss and S. Arbanowski, "Social Preference Ontologies for Enriching User and Item Data in Recommendation Systems," in IEEE Int. Conf. on Data Mining Workshop, 2014, pp. 365-372.

[16] C. M. Bastemeijer, L. Voogt, J. P. van Ewijk, and J. A. Hazelzet, "What do patient values and preferences mean? A taxonomy based on a systematic review of qualitative papers," Patient Education and Counseling, 122016. 


\begin{tabular}{|c|c|c|c|}
\hline Category & Primary & Modifier & Textual definition \\
\hline Speed & Medium & & "I generally don't want the robot to move fast nor slow" \\
\hline $\begin{array}{c}\text { Information } \\
\text { Providing }\end{array}$ & Always & & "I prefer that the robot talks to me" \\
\hline Petitions & Minimum & & "I prefer the robot to assist me without bothering" \\
\hline $\begin{array}{c}\text { Social } \\
\text { Behavior }\end{array}$ & Informal and funny & & "I like robots that make jokes" \\
\hline $\begin{array}{c}\text { Social } \\
\text { Behavior }\end{array}$ & Formal and polite & $\operatorname{not(Company/None)}$ & "I want the robot to be polite when I am not alone" \\
\hline
\end{tabular}

TABLE I: Example of Aunt Mery's global (task independent) preferences.

\begin{tabular}{|c|c|c|c|}
\hline Category & Primary & Modifier & Textual definition \\
\hline \multirow{4}{*}{ Motor } & Right arm first & & "The right arm is injured so it's easier to put it first" \\
\hline & Both arms together & Company/Caregiver & $\begin{array}{c}\text { "With the help of the caregiver it's easier to put on both } \\
\text { arms together" }\end{array}$ \\
\hline & Lateral trajectory & & $\begin{array}{c}\text { "I like it more when the coat dressing is started from } \\
\text { one side" }\end{array}$ \\
\hline & Start position low & & $\begin{array}{c}\text { "The robot should start from a low position for easier } \\
\text { dressing" }\end{array}$ \\
\hline \multirow{2}{*}{ Force } & Low & Moment/Putting the right sleeve & "The injured arm can't take much pressure" \\
\hline & Normal & & $\begin{array}{c}\text { "I prefer the robot not to use too much force when } \\
\text { dressing me" }\end{array}$ \\
\hline
\end{tabular}

TABLE II: Examples of Aunt Mery's preferences for the coat dressing task.

\begin{tabular}{|c|c|c|c|}
\hline Category & Primary & Modifier & Textual definition \\
\hline Speed & Slow* & Moment/Night & $\begin{array}{c}\text { "Prefer to fit the shoe slower at night as I am more } \\
\text { tired" }\end{array}$ \\
\hline \multirow{3}{*}{ Motor } & Left foot first & & "It's more comfortable to put on the left foot first" \\
\hline & Straight foot & & "I don't feel comfortable with the foot turned" \\
\hline & Left approach & & "It's better if the robot approaches for the left" \\
\hline Petitions & Sometimes* & Company/Caregiver & $\begin{array}{c}\text { "The Caregiver helps me understand the robot and how } \\
\text { to reposition myself" }\end{array}$ \\
\hline $\begin{array}{l}\text { Information } \\
\text { providing }\end{array}$ & None* & Company/Caregiver & "The caregiver already gives me enough information" \\
\hline \multirow[t]{2}{*}{ Temporal } & 30 sec. lifted & & $\begin{array}{c}\text { "I can't hold the foot lifted for much time when I'm } \\
\text { alone" }\end{array}$ \\
\hline & 1 min. lifted & Company/Family member & "They help me hold on with the lifted foot" \\
\hline
\end{tabular}

TABLE III: Examples of Aunt Mery's preferences for the shoe fitting task.

\begin{tabular}{|c|c|c|c|}
\hline Category & Primary & Modifier & Textual definition \\
\hline Speed & Slow* & Moment/Morning & "I like to take my breakfast calmly" \\
\hline $\begin{array}{c}\text { Motor/ } \\
\text { Proxemic }\end{array}$ & Outside feed & & $\begin{array}{l}\text { "I can't move the spoon but I don't need the robot to } \\
\text { insert it in my mouth" }\end{array}$ \\
\hline \multirow{3}{*}{ Motor } & Straight scooping & & $\begin{array}{l}\text { "I don't want the robot to move much when I'm biting } \\
\text { the spoon" }\end{array}$ \\
\hline & Left-side approach & Situation/Kitchen & $\begin{array}{l}\text { "I'm more comfortable when the robot is in the left } \\
\text { side" }\end{array}$ \\
\hline & Right-side approach & Situation/Dining room & $\begin{array}{l}\text { "In the dining room I feel better when the robot is in } \\
\text { my right side" }\end{array}$ \\
\hline $\begin{array}{l}\text { Personal } \\
\text { tastes }\end{array}$ & Low temperature & & "I prefer to wait until the food is cooler" \\
\hline $\begin{array}{l}\text { Information } \\
\text { providing }\end{array}$ & High* & Company/None & $\begin{array}{l}\text { "I feel more accompanied when the robot talks while } \\
\text { eating alone" }\end{array}$ \\
\hline $\begin{array}{c}\text { Information } \\
\text { obtaining }\end{array}$ & Only when needed & & "I don't like to answer questions while eating" \\
\hline Cognitive & Remind after lunch pills & Moment/Afternoon & "I don't want to forget to take my medicine" \\
\hline
\end{tabular}

TABLE IV: Examples of Aunt Mery's preferences for the feeding task.

[17] J. M. Beer, A. D. Fisk, and W. A. Rogers, "Toward a Framework for Levels of Robot Autonomy in Human-Robot Interaction," Journal of Human-Robot Interaction, vol. 3, no. 2, p. 74, 2014.

[18] H. Peng, C. Zhou, H. Hu, F. Chao, and J. Li, "Robotic dance in social robotics - A taxonomy," IEEE Transactions on Human-Machine Systems, vol. 45, no. 3, pp. 281-293, 2015.

[19] J. Shim and R. C. Arkin, "A Taxonomy of Robot Deception and Its Benefits in HRI," in 2013 IEEE Int. Conf. on Systems, Man, and Cybernetics. IEEE, 10 2013, pp. 2328-2335.

[20] T. J. Wiltshire, E. J. C. Lobato, J. Velez, F. G. Jentsch, and S. M. Fiore, "An interdisciplinary taxonomy of social cues and signals in the service of engineering robotic social intelligence," in Proc. SPIE, vol. 9084, 62014 .
[21] T. Fong, I. Nourbakhsh, and K. Dautenhahn, "A survey of socially interactive robots," Robot. Auton. Syst., vol. 42, no. 3-4, pp. 143-166, 2003.

[22] M. Tenorth and M. Beetz, "KnowRob: A knowledge processing infrastructure for cognition-enabled robots," The Int. Journal of Robotics Research, vol. 32, no. 5, pp. 566-590, 2013.

[23] O. P. John and S. Srivastava, "The big five trait taxonomy: History, measurement, and theoretical perspectives," Handbook of personality: Theory and research, vol. 2, no. 1999, pp. 102-138, 1999.

[24] C. LeRouge, J. Ma, S. Sneha, and K. Tolle, "User profiles and personas in the design and development of consumer health technologies," Int. Journal of Medical Informatics, vol. 82, no. 11, pp. e251 - e268, 2013. 